\title{
Subtypes of Intellectual Disability in School-Aged Children
}

\author{
Andrew Taylor ${ }^{1,2,}$, , Cory Saunders ${ }^{2}$, Nikhil S. Koushik ${ }^{2}$, John Strang ${ }^{2}$ and Joseph Casey ${ }^{2,3}$ \\ ${ }^{1}$ The Windsor Essex Community Health Centre, Windsor, Ontario, Canada \\ ${ }^{2}$ Ozad Institute, Windsor Regional Children's Centre, Windsor, Ontario, Canada \\ ${ }^{3}$ Department of Psychology, University of Windsor, Windsor, Ontario, Canada
}

\begin{abstract}
Purpose: To explore the presence of subtypes of intellectual functioning in children with mild intellectual disability (ID) and to externally validate the subtypes on measures of academic, adaptive and psychosocial functioning.

Method: Participants were 167 children age 6-16 years with a mild ID. All children completed the WISC-III, WIAT, VABS, and PIC-R.

Results: Based on a two-stage cluster analysis on the four WISC-III Index scores four subtypes emerged reflecting distinct profiles: (1) language-strength subtype; (2) nonverbal strength subtype; (3) symbol-processing strength subtype; and (4) global deficits subtype. The subtypes were externally validated on tests of academic achievement, adaptive functioning, and psychosocial functioning.

Conclusions: Rather than only demonstrating a global or "flat" pattern of cognitive deficits, the findings suggest that children with mild ID have certain profiles of intellectual functioning that are similar to those seen in normal children and children with learning disabilities.
\end{abstract}

Keywords: Intellectual disability, cognitive strengths, children, neurodevelopmental disorders.

Although many investigations of children with learning disabilities and with normal children have revealed distinct subtypes based on intellectual variables (e.g., [1-3]), little research has focused on examining the presence of subtypes of children with intellectual disability (ID) ${ }^{1}$ based on their level and pattern of cognitive competencies, despite clinical evidence that these children differ in meaningful ways. Nonetheless, a number of research studies conducted in the last decade revealed distinct subtypes of children with ID [4] have been identified on variables ranging from communication competencies [5], displays of empathy [6, 7], levels of psychopathology [8], to specific cognitive test performances [9].

\section{Patterns of Intellectual Functioning Using the Wechsler Intelligence Scale for Children-Third Edition (WISC-III)}

A substantial amount of research exists supporting the presence of distinct cognitive profiles on tests of intelligence in normative groups of children and children with learning disabilities. Konold, et al. [1] examined the cognitive profiles of the WISC-III [10]

*Address correspondence to this author at the Windsor Essex Community Health Centre, Windsor, Ontario, N8X 1K5, Canada; Tel: 519-253-8481; Fax: 519-253-4362; E-mail: ataylor@wechc.org

\footnotetext{
${ }^{1}$ Where appropriate, the contemporary term "intellectual disability" is used throughout instead of mental retardation for the reasons described in the definitional manual of the AAIDD (2010).
}

standardization sample using cluster analysis. Eight distinct profiles were reported: a high WISC-III FSIQ (Full Scale IQ) group, an above average FSIQ group, an above average $\mathrm{VIQ}$ (Verbal IQ) $>\mathrm{PIQ}$ (Performance $I Q)$ group, an average $P I Q>V I Q$ group, a below average $P I Q>V I Q$ group, and a generally low ability FSIQ group. Cluster analytic research utilizing the WISC-III completed by Donders [11] involved analysis of 2200 WISC-III profiles from a normal distribution of children. The results of this study revealed five distinct clusters. Three of the groups were based on overall level of performance (i.e., above average on all four WISC-III Index scores, average on all Index scores, and below average on all Index scores). There was also a high Processing Speed group and a low Processing Speed group. In contrast, Saunders et al. [2] reported six clusters in their analysis of a heterogeneous sample of clinic-referred children who had completed the WISC-III as part of a comprehensive neuropsychological assessment, some of which were distinct from the subtypes found by Konold et al. and Donders. Clusters included groups of children with broad based processing difficulties, deficient language abilities, deficient nonverbal abilities, deficits consistent with a WISC-III ACID (relative weaknesses on Arithmetic, Comprehension, Information, and Digit Span subtests) pattern, deficient working memory, and deficits in tasks involving visual sequencing and language abilities. 


\section{Evidence for Subtypes Based on the Vineland Adaptive Behavior Scales (VABS)}

The conceptualization of ID has evolved over the years. Although there was some recognition of the importance of adaptive behaviors, or problems with maturation and social adjustment, the diagnosis of before the early 1970s was based almost exclusively on measured intelligence [12]. The importance of adaptive behavioral functioning increased when the American Association of Mental Deficiency (AAMD) made concurrent measured deficits in adaptive behavior and sub-average intellectual functioning a requirement for the diagnosis of Mental Retardation in 1973 [12]. Since the inclusion of adaptive behavior deficits in the diagnosis of ID, there has been substantial debate in the literature about its definition and appropriate measurement. Although this debate remains ongoing, the assessment of adaptive behaviors in children with ID was made easier with the introduction of the Vineland Adaptive Behavior Scales (VABS [13]). The VABS enables the systematic assessment of adaptive behavior through the administration of scales to an informant (typically a parent or primary caregiver) who is asked to describe the child's everyday behavior in four domains: Communication, Daily Living, Socialization, and Motor Skills. Standard scores and age equivalents are calculated for each domain. These domain scores are then combined to yield an adaptive behavior composite score. The VABS has excellent psychometric properties and is regarded as one of the foremost psychometric measures of adaptive behavior [12].

Despite the importance of concurrent intellectual and adaptive behavior deficits for the diagnosis of Intellectual Disability, there is a paucity of research in the literature addressing the relationship between these two constructs. In one such investigation, Carpenteri and Morgan [14] examined the relationship between adaptive functioning on the VABS and intellectual functioning on the Stanford-Binet Intelligence Scale, $4^{\text {th }}$ edition (SB-IV). Their sample consisted of a group of children with autism and co-existing ID and a group of non-autistic children with ID. The two groups were of comparable chronological age and FSIQ. The results indicated that only 5 of the 20 correlations between the various domains of the two instruments were significant for the group of non-autistic children with ID. Moreover, all five of the significant correlations were between the various ability domains of the SB-IV and the Communication domain of the VABS. Whereas the correlation between the overall composite scores for the two measures for children with autism and ID was .77 , it was only .29 for the non-autistic children with ID.

Other research adds to, and serves to clarify, the significance of the findings of Carpenteri and Morgan [14]. Liss, et al. [15] examined the relationship between adaptive functioning, psychometric intelligence, autistic symptomatology, and cognitive tests of language and memory. To meet this objective they compared children with high-functioning autism to age-matched children with developmental language disorders, as well as lowfunctioning children with autism to age-matched children with low scores on tests of adaptive functioning, IQ, and tests of language and verbal memory. They labeled children in the last group as their "low IQ or Mental Retardation" group. Groups were compared with regard to the Communication, Socialization, and Daily Living Skills domains of the VABS. Moderate to high correlations were reported between the three IQ composites of the SB-IV and domains of adaptive functioning on the VABS for the low IQ group. Specifically correlations ranged from .39 -.90 across skill areas with a mean of .60. Verbal intelligence correlated more strongly with all three VABS domains than non-verbal intelligence. Nonverbal intelligence was significantly correlated with only the Communication and Socialization domains. These authors note that the group labeled "low IQ or Mental Retardation" may have been significantly heterogeneous in nature, noting that in addition to low scores on measures of adaptive functioning and psychometric intelligence, some of the children in this group exhibited minor autistic behaviors. As a result, the generalization of their findings to other groups of children with ID may be limited.

Considering these two investigations, it is important to note that there were differences in Verbal, Nonverbal, and Quantitative IQ scores for the children with ID in the two samples. Standard scores on these three composites were 64,53 , and 58 respectively for the Carpenteri and Morgan investigation, whereas they were 55, 55, and 32 respectively for the Liss et al. [15] investigation. Liss et al. suggested the possibility that scores on psychometric measures of intelligence may be more highly correlated with psychometric measures of adaptive behavior at lower levels of functioning. There is some evidence in the literature to support this view $[16,17]$. It is possible that at low levels of global functioning, both psychometric intelligence and adaptive behavior are measuring similar constructs, involving perhaps the ability to understand and master simple rule-governed tasks. 


\section{Evidence for Subtypes Based on the Wechsler Individual Achievement Test (WIAT)}

There is research involving the correlation of academic achievement patterns with intellectual profiles. The technical manual of the WIAT includes a correlation between the FSIQ of the WISC-III and the Total Achievement score of the WIAT, which is between 0.30 and 0.70 for children ages six to sixteen [18]. Waxman and Casey [3] completed a cluster analytic study utilizing both the WISC-III and the WIAT in a population of children referred for neuropsychological assessment because of concerns regarding their academic achievement, behavioral functioning, or both. The findings from this study supported a five-cluster solution which included a group with predominantly low ability and achievement, a group with verbal processing deficits, a group demonstrating visual spatial/processing speed deficits, a group with generally low ability and achievement and average processing speed, and a group with deficits consistent with a WISC-III ACID pattern.

\section{Evidence for Subtypes Based on the Personality Inventory for Children Revised (PIC-R)}

Subtypes of children who exhibit intellectual deficits have been identified on measures of emotional and behavioral adjustment. Ralston, et al. [8] completed a Q-factor analysis of Personality Inventory for Children (PIC) scores on 101 children with intellectual deficits and Full Scale IQ scores ranging from 60 to 84 . The analyses demonstrated several distinct psychosocial subtypes, including normal (absence of clinically relevant psychopathology), internalized psychopathology, somatic concern, mild hyperactive, and mild anxiety/depression. The behavioral subtypes identified by these authors matched those found in a population of children with learning disabilities and normal range IQs [19].

Nunn, Tonge, and Einfeld [20] also identified subtypes of children with ID on psychosocial variables. This investigation employed cluster analysis to a group of 558 participants with ID using six scales from the Developmental Behaviour Checklist. The results of this study identified three distinct subtypes: children with good psychosocial adjustment, children with high levels of mixed internalizing and externalizing symptomatology, and children with a predominance of symptoms with "self-absorbed and autistic-style" dimensions.

Kasari et al. [6] conducted a study examining the empathic ability of 30 children with Down syndrome, 22 children with nonspecific etiologies of ID, and 22 normally developing children. The results of this study showed that the Down syndrome group responded to distress with increased eye contact and empathic support in comparison with the other two groups of children.

In summary, research with healthy controls and children with learning disabilities has revealed distinct subtypes of children based on their level and pattern of intellectual differences. In particular, there appears to be convergent evidence supporting the presence of intellectual subtypes characterized by: (1) better developed verbal skills, (2) stronger nonverbal skills, (3) average capabilities in all areas, and (4) deficits in all areas. There have also been a number of less wellsupported profiles identified characterized by more specific strengths or weaknesses (e.g., freedom from distractibility/working memory, visual symbol processing speed).

\section{Hypotheses}

Research in the area of ID has identified distinct subtypes within this population on a number of behavioral and emotional variables. There has also been indication of possible cognitive processing differences [11]. Since cluster analytic research with normative and learning disability populations have consistently revealed distinct subtypes of children based on cognitive abilities, it was hypothesized that children with ID would also exhibit different patterns of cognitive abilities that could be identified through cluster analysis.

Given that research investigations have reported similar groupings of children in learning disabled population and borderline/intellectually deficient populations based on IQ scores (e.g., Ralston et al., [8]), it was hypothesized that previously reported cognitive subtypes identified in normal and clinical populations would also be found in children with ID. However, due to decreased variability in IQ scores of children with ID, it was also expected that fewer subtypes would be identified and that identified subtypes would represent the most robust patterns of cognitive performance previously identified (e.g., language deficits, nonverbal deficits). Finally, in an effort to have a comprehensive understanding of children with ID and in acknowledgement that children with ID often are impacted in their adaptive functioning, academic functioning, and are at greater risk for psychopathology, it was hypothesized that identified 
subtypes would also be represented across academic domains, adaptive domains, and patterns of psychopathology. Better characterizing patterns of intellectual, adaptive and socio-emotional functioning in children with mild IDs, regardless of etiology, is imperative to long-term academic and treatment planning.

\section{METHOD}

\section{Participants}

The sample consisted of 167 children and adolescents (102 boys, 65 girls) between the ages of 6 and 16 years $(M=10.61, S D=2.27)$ who had been diagnosed with ID. These included children with a WISC-III Full Scale IQ of 75 or lower with concomitant deficits in adaptive behavior. The decision to include children with IQs between 70 and 75 was based on the criteria for Mental Retardation in DSM-IV [4], which has been most recently supported by the American Association on Intellectual and Developmental Disability [21] in their definition of intellectual disability. This decision to include children with IQs between 70 and 75 who have concomitant deficits in adaptive behavior, was further supported by consideration of the Flynn Effect, as described by Kanaya, Scullin and Ceci [22]. These authors pointed out that when IQ tests are periodically revised and re-normed, they become more stringent in terms of age-appropriate expectations. As a result, longitudinal IQ records demonstrated that students in the Borderline and Mild ID range lost an average of 5.6 points when they were re-tested on a renormed Wechsler IQ test, increasing the likelihood of their classification in the ID range.

Most of the participating children in our sample had nonspecific etiologies for their ID. A smaller portion of the sample included children with a variety of identifiable conditions, including genetic or teratologic disorders (e.g., Fragile X, Down Syndrome, Fetal Alcohol Syndrome), neurological disorders (e.g., cerebral palsy, epilepsy), and other neurodevelopmental disorders (e.g., high functioning autism or PDD). Participants had been referred to a regional children's mental health center in Southwestern Ontario for neuropsychological assessment due to a combination of known or suspected underlying learning disorders, combined with parent reports of behavioral, emotional, or social difficulties at the time of case intake. All children were administered the WISC-III, WIAT-II, and the PIC-R, however due to clinical need and referral information only 86 of the children in our sample were administered the VABS at the time of assessment.

\section{Measures}

Morris, Blashfield, and Satz [23], defined cluster analysis as a multivariate procedure for detecting relatively homogenous groupings among subjects. Hierarchical methods and iterative partitioning methods of cluster analysis were employed in the present study. Hierarchical clustering procedures consider all possible combinations of clusters and combine clusters which serve to minimize the increase in the error sum of squares [23]. Iterative procedures (typically utilizing the $k$-means analysis method), as described by Morris et al. [23], involves estimate of cluster centroids followed by the assignment of subjects to the clusters with the most similar centroids. The process is repeated iteratively until a stable solution can be identified. As was reported by Fuerst and Rourke [24], iterative partitioning methods are well suited to clustering large data sets. Cluster analysis has been used in a number of studies examining intellectual competency differences in general, and among children specifically (e.g., [1-3, 11, 25-27]). There has been good support for using the Ward's method as a hierarchical procedure (e.g., $[25,24,28]$ ). Use of the $k$-means iterative portioning procedure has also found support in the representative child cluster analysis literature (e.g., [29]; see also [2, 24, 30]).

\section{Procedure}

To address potential issues related to underlying genetic or other neurodevelopmental disorders that may be associated with intellectual subtypes, patient charts were reviewed to identify the presence or absence of previous neurodevelopmental diagnoses or previous genetic testing. Although the majority of participants did not have previous genetic testing, in many cases a genetic cause was ruled out. In contrast, $13.9 \%$ of the sample had experienced pre/perinatal insults and $42 \%$ had experienced one or more other medical disorders. As a result, no relationship was found between underlying genetic or neurodevelopmental conditions and subtype assignment. These data are summarized in Table 1.

\section{Statistical Analysis}

To determine the most viable clinical subtypes that existed in the data, a two-stage cluster analysis was applied to the four WISC-III Factor Index scores (e.g. 
Table 1: Prevalence of Risk Variables in Total Sample and WISC-III Subtypes ${ }^{a}$

\begin{tabular}{|c|c|c|c|c|c|c|}
\hline & & $\begin{array}{c}\text { Total } \\
\text { Sample } \\
(n=168)\end{array}$ & $\begin{array}{l}\text { VPS } \\
(n=35)\end{array}$ & $\begin{array}{c}\text { NPS } \\
(n=50)\end{array}$ & $\begin{array}{c}\text { PSS } \\
(n=31)\end{array}$ & $\begin{array}{c}\text { LIA } \\
(n=52)\end{array}$ \\
\hline \multicolumn{7}{|c|}{ Family Environment } \\
\hline & Parental drug use & 12.0 & 5.7 & 14.6 & 19.4 & 9.6 \\
\hline \multicolumn{2}{|r|}{ Poverty $(<\$ 20,000 /$ year $)$} & 13.3 & 11.4 & 22.9 & 3.2 & 11.5 \\
\hline \multicolumn{2}{|r|}{ Parent < grade 12 educational attainment } & 13.3 & 20.0 & 12.5 & 16.1 & 7.7 \\
\hline \multicolumn{2}{|r|}{ Parent(s) unemployed } & 10.2 & 0.0 & 18.8 & 12.9 & 7.7 \\
\hline \multicolumn{2}{|r|}{ Child Abuse/neglect } & 30.1 & 28.6 & 35.4 & 29.0 & 26.9 \\
\hline \multicolumn{7}{|c|}{ Child Medical Concerns } \\
\hline \multicolumn{2}{|r|}{ Lack of prenatal care } & 1.2 & 0.0 & 2.1 & 0.0 & 1.9 \\
\hline \multicolumn{2}{|r|}{ Teratogens } & 13.3 & 11.4 & 10.4 & 19.4 & 13.5 \\
\hline \multicolumn{2}{|r|}{ LBW or premature } & 13.3 & 17.1 & 10.4 & 16.1 & 11.5 \\
\hline \multicolumn{2}{|r|}{ History of pre/perinatal insult } & 13.9 & 8.6 & 10.4 & 16.1 & 19.2 \\
\hline & Hypoxia/ischemia & 10.2 & 2.9 & 6.3 & 12.9 & 17.3 \\
\hline & Unspecified brain injury & 1.8 & 2.9 & 2.1 & 0.0 & 1.9 \\
\hline & Other & 1.2 & 2.9 & 2.1 & 0.0 & 0.0 \\
\hline \multicolumn{2}{|r|}{ Has medical disorder } & 42.2 & 54.3 & 31.3 & 41.9 & 44.2 \\
\hline \multicolumn{7}{|c|}{ Genetic Testing } \\
\hline & None conducted & 81.9 & 85.7 & 85.4 & 77.4 & 78.8 \\
\hline & Referred but no results provided & 3.0 & 0.0 & 6.3 & 3.2 & 1.9 \\
\hline & Genetic Disorder Identified & 3.6 & 2.9 & 2.1 & 3.2 & 5.8 \\
\hline & Results indicated no genetic disorder & 11.4 & 11.4 & 6.3 & 16.1 & 13.5 \\
\hline \multicolumn{7}{|c|}{ Child Psychiatric Concerns } \\
\hline \multicolumn{2}{|r|}{ Has psychiatric disorder } & 39.2 & 31.4 & 50.0 & 41.9 & 32.7 \\
\hline \multicolumn{2}{|r|}{ History of outpatient treatment } & 56.6 & 62.9 & 62.5 & 61.3 & 44.2 \\
\hline \multicolumn{2}{|r|}{ History of inpatient/residential treatment } & 15.1 & 11.4 & 12.5 & 19.4 & 17.3 \\
\hline \multicolumn{2}{|r|}{ Taking psychotropic medication } & 39.2 & 28.6 & 52.1 & 32.3 & 38.5 \\
\hline \multicolumn{2}{|c|}{ Enrolled in Special Education } & 83.9 & 77.1 & 87.5 & 83.9 & 88.5 \\
\hline
\end{tabular}

${ }^{a}$ valid percents reported.

Verbal Comprehension, Perceptual Organization, Freedom from Distractibility, and Processing Speed). This two-stage cluster analysis followed a procedure described in Jones, Drummond, Saunders, and Strang [31]. In the first stage, a hierarchical cluster analysis was conducted using the Mean Centroid method of group linkage. Squared Euclidean distance was chosen as a measure of similarity, because it preserves the shape, elevation, and scatter of the data [32]. To correct for fusion errors resulting from the hierarchical clustering technique, the mean centroids were used as seed values for the iterative partitioning method conducted during the second stage. This $k$-means analysis was performed for each solution that appeared clinically valid. The final cluster centers were calculated for the mean profiles for each subtype to examine their clinical relevance. For the purposes of reliability this method was repeated using between group linkage with $k$-means correction. To determine the reliability of the final cluster solutions using both methods cluster membership was compared using Goodman and Kruskal's tau, a measure of similarity for nominal variables.

\section{RESULTS}

\section{General Sample Findings}

Examination of the overall sample revealed a mean WISC-III FSIQ of 63.32 (SD = 9.04). The resulting 
Mean WISC-III Factor Scores for the Entire Sample

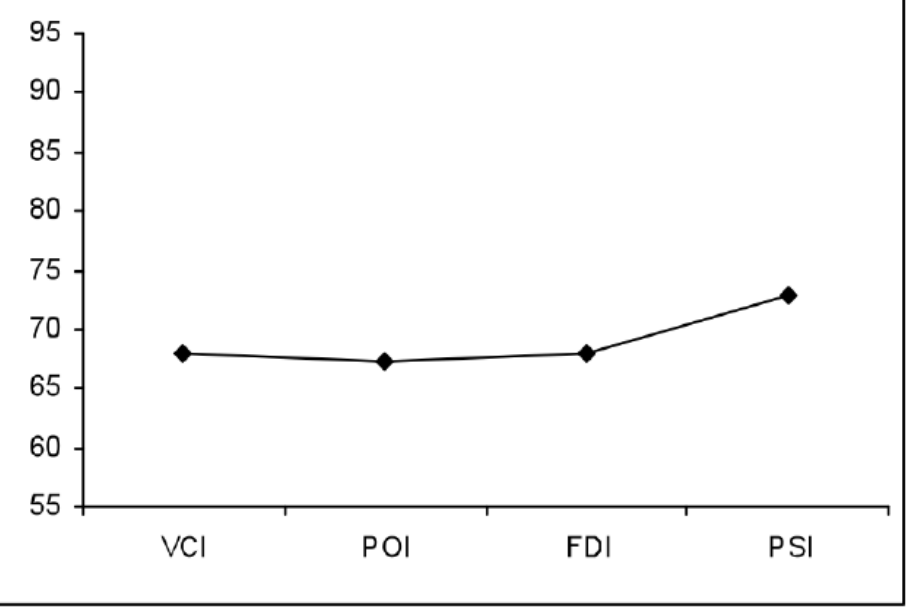

Figure 1: Mean WISC-III Factor Scores for Entire Sample.

profile of factor indexes was relatively flat, with scores ranging from 67.50 to 73.40 . The overall factor index pattern is depicted in Figure 1.

\section{Cluster Analysis}

When the WISC-III Factor Index scores were analyzed, the resultant agglomeration schedule coefficients and dendrograms for the hierarchical cluster analysis yielded a four-cluster solution. These clusters were subsequently subjected to an iterative partitioning method using $k$-means analysis $(k=4)$. The clusters appeared to represent distinct patterns of cognitive ability. The mean Factor Index scores for each cluster are presented in Table 2. Level of association between the cluster solutions using the mean centroid and between group linkage-based subtypes within the sample was examined using Goodman and Kruskal's tau. Data analysis yielded a statistically significant level of association between subject membership in the clusters generated using the two different methods of the present study $(\tau=.863$, $p<.000)$.
The clusters identified in this analysis demonstrated the following mean subtype patterns that were described in terms of most salient WISC-III characteristics: language processing strengths (LPS), visual motor (or nonverbal processing) strengths (NPS), processing speed strengths (PSS), and global deficits (GD). The children with language processing strengths evidenced elevations on the WISC-III Verbal Comprehension and Freedom from Distractibility Index scores, the latter Index emphasizing auditory-verbal attention and short-term memory. The visual motor strengths subtype had elevations on Perceptual Organization and Processing Speed Indexes. The processing speed strengths subtype showed markedly elevated Processing Speed Index, with a secondary elevation on Freedom from Distractibility Index. The final subtype showed a WISC-III Index score pattern with no significant elevations, consistent with global deficits.

\section{Academic Achievement Characteristics}

To examine differences in achievement patterns based on the above identified intellectual subtypes, a

Table 2: Mean WISC-III Factor Scores and Standard Deviations for ID Subtypes

\begin{tabular}{|c|c|c|c|c|}
\hline & VPS & NPS & PSS & GD \\
\hline Verbal Comprehension & $79.30(6.40)^{\mathrm{a}}$ & $67.60(8.90)$ & $72.30(7.40)$ & $59.83(6.69)$ \\
\hline Freedom From Distractibility & $80.70(7.70)$ & $65.14(8.70)$ & $77.80(10.9)$ & $58.00(7.56)$ \\
\hline Processing Speed & $74.90(7.90)$ & $71.70(8.80)$ & $96.20(7.90)$ & $62.29(9.26)$ \\
\hline
\end{tabular}

${ }^{\mathrm{a}}$ Numbers in parentheses are standard deviations. 
Table 3: Mean WIAT Scores and Standard Deviations by ID Subtypes

\begin{tabular}{|c|c|c|c|c|}
\hline & LPS & NPS & PSS & GD \\
\hline \hline Basic Reading & $79.86(13.85)^{\mathrm{c}}$ & $73.45^{\mathrm{a}}(13.87)$ & $84.68(14.96)$ & $70.14(11.53)$ \\
\hline Math Reasoning & $74.56(7.96)$ & $68.29^{\mathrm{b}}(8.63)$ & $79.74(15.50)$ & $64.53(7.91)$ \\
\hline Spelling & $77.77(12.61)$ & $72.25(12.56)$ & $79.89(12.35)$ & $68.53(9.50)$ \\
\hline Numerical Operations & $72.08(9.20)$ & $67.53(9.26)$ & $76.33(14.94)$ & $61.18(8.55)$ \\
\hline
\end{tabular}

${ }^{a}$ NPS group significantly lower than PSS group when FSIQ is controlled.

${ }^{b}$ NPS group significantly lower than all other groups when FSIQ is controlled.

${ }^{\mathrm{c}}$ Numbers in parentheses are standard deviations.

MANCOVA controlling for FSIQ was conducted on four subtests of the WIAT including: Basic Reading, Spelling, Math Reasoning, and Numerical Operations. The results of this analysis revealed academic profile differences between the four ID subtypes $(F=2.487$, $p<.01)$. Subsequent individual ANCOVAs revealed ID subtype differences for Basic Reading $(F=3.362$, $p<.05)$, Math Reasoning $(F=6.954, p<.001)$, and Numerical Operations $(F=3.496, p<.05)$. The overall factor index pattern as related to academic achievement is presented in Table 3.

\section{Adaptive Functioning}

The Pearson correlation between the VABS Adaptive Behavior Composite (ABC) score and WISCIII FSIQ was statistically significant only for the children with the lowest FSIQ, namely the children in the global deficits subtype, $r=.320(p<.05)$. Further analyses revealed that for the children in the global deficits subtype, the Pearson correlation between VIQ and the VABS ABC score was significant $r=.487,(p<.05)$ whereas that between PIQ and the VABS ABC was not $r=.247$.

The four ID subtypes were differentiated on the VABS. There were significant differences in the VABS $A B C$ scores across subtypes, even after controlling for the effects of $\mathrm{FSIQ}, F(4,81)=2.89, p<.05$. Subsequent analysis controlling for the effects of FSIQ revealed significant statistical differences between some of the four ID subtypes on the Daily Living Skills (DLS) domain, $F(3,81)=3.29, p<.05$. The DLS score of children with global deficits was significantly lower than that of children with language strengths or symbol processing strengths. The DLS score of children with visual-motor strengths was significantly lower than that of children with symbol processing strengths. The mean VABS profiles for each of the four intellectual functioning subtypes of children with ID are presented in Table 4

\section{Psychosocial Attributes}

When the PIC-R scores were cluster analyzed, the resultant agglomeration schedule coefficients and dendrograms for the hierarchical cluster analysis yielded a three-cluster solution. These clusters were subsequently subjected to an iterative partitioning method using $k$-means analysis $(k=3)$. The clusters appeared to represent distinct patterns of psychopathology.

The clusters identified in this analysis demonstrated the following mean subtype patterns that were described in terms of most salient psychopathology characteristics as follows: No Psychopathology, Internalized Psychopathology, and Mixed Psychopathology. Of the children with language processing strengths, $50 \%$ fell in the No Psychopathology group, $20 \%$ fell in the Internalized Psychopathology group, and $30 \%$ fell in the Mixed

Table 4: Mean VABS Scores and Standard Deviations in WISC Subtypes

\begin{tabular}{|c|c|c|c|}
\hline & LPS & NPS & GD \\
\hline \hline Communication & $59.80(9.04)^{\mathrm{a}}$ & $56.00(15.19)$ & $49.38^{\mathrm{b}(13.65)}$ \\
\hline Daily Living Skills & $58.20(9.85)$ & $52.60(14.41)$ & $47.60(14.81)$ \\
\hline Socialization & $61.00(11.43)$ & $60.75(16.03)$ & $56.86(12.60)$ \\
\hline
\end{tabular}

Note. Processing Speed Strength group was not included due to low cell size.

${ }^{a}$ Numbers in parentheses are standard deviations.

${ }^{\mathrm{b}} \mathrm{GD}$ group is significantly lower than LPS group. 


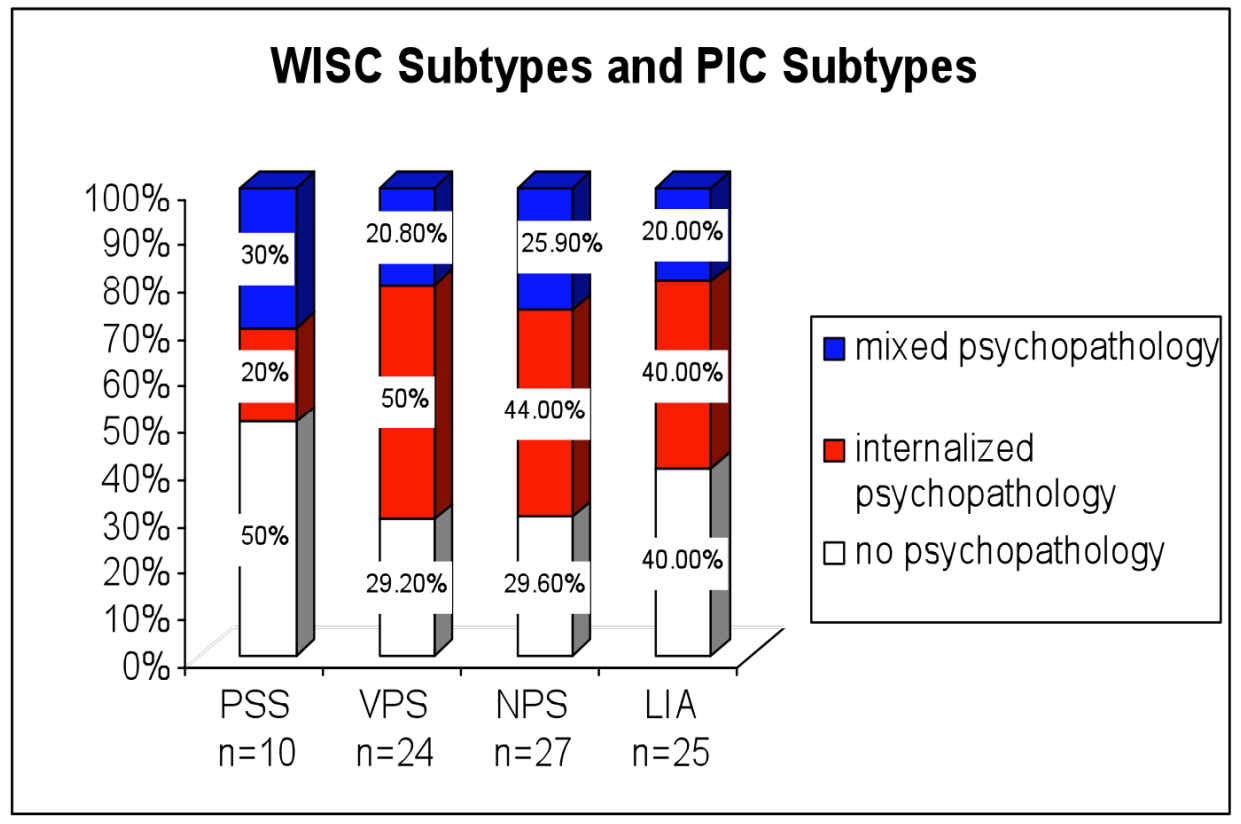

Figure 2: Percentage of PIC subtypes in WISC subtypes.

Psychopathology group. Of the visual motor strengths subtype $29.6 \%$ fell in the No Psychopathology group, $44 \%$ fell in the Internalized Psychopathology group, and $25.9 \%$ fell in the Mixed group. The symbol processing strengths subtype revealed $50 \%$ in the No Psychopathology group, $20 \%$ in the Internalized Psychopathology group, and $30 \%$ in the Mixed Psychopathology group. The final, Low in All subtype showed $40 \%$ in the No Psychopathology group, $40 \%$ in the Internalized Psychopathology group, and $20 \%$ in the Mixed Psychopathology group. Each of these cluster subtypes is depicted in Figure 2.

\section{DISCUSSION}

The hypothesis that distinct subtypes of cognitive functioning would be found for children with ID was supported. Four distinct subtypes were found, the profiles of which have been identified in previous investigations of normally developing and learning disabled populations (i.e., [1-3]). The subtypes of children with ID included the following patterns: symbol processing strengths, language-related strengths, nonverbal strengths, and children with global deficits or homogeneity of intellectual deficiencies. The findings demonstrated that intellectual subtypes exists in a population of children diagnosed with a mild ID, and that some are similar in pattern (not level) to those identified in normative and LD populations. The identification of intellectual subtypes of ID also fits with other studies that identified additional patterns of strengths within this population (i.e., $[6,20]$ ).
Intellectual subtypes of ID were not evident in this sample when the WISC Full Scale IQ was at the lower end of the measurable range based on the standardization data. While the latter finding differs from research with normal and LD populations, it is consistent with the general rule that we are less likely to see true differences in intellectual strengths and weaknesses on IQ tests at the extreme ends of the population distribution largely due to inherent ceiling and (in this case) floor effects of "intelligence" tests. Also, the failure to identify intellectual subtypes of ID for the children in our study sample who were especially lower functioning supported the long-held position in the literature that there is a meaningful difference between individuals with DSM-IV diagnosed Mild Mental Retardation and those diagnosed with DSM-IV Moderate Mental Retardation (e.g., see Diagnostic and Statistical Manual of Mental Disorders, Fourth Edition: Text Revision [4]).

The following includes a description of findings associated with each identified subtype ID.

\section{Processing Speed Strengths}

This subtype of children demonstrated markedly elevated WISC-III Processing Speed Index, with a secondary elevation on Freedom from Distractibility. In addition, this group demonstrated higher academic achievement, particularly in WIAT Basic Reading. Further, this group demonstrated higher scores on the Daily Living Scale of the VABS in comparison with the GD group and the NVS group. Fifty percent of these 
children exhibited significant psychopathology on the PIC in the form of either Internalized Psychopathology $(20 \%)$ or Mixed Psychopathology (30\%).

\section{Language Processing Strengths}

This subtype of children demonstrated markedly elevated Verbal Comprehension, with a secondary elevation on Freedom from Distractibility. In addition, this group demonstrated higher overall academic achievement compared to the other subtypes, as well in WIAT Basic Reading, although the mean Basic Reading score for the LPS group was lower when compared to the PSS group. The LPS group demonstrated higher scores on the Communication Scale of the VABS in comparison with the GD group. Similar to the PSS group, $50 \%$ of these children exhibited significant psychopathology of the PIC, with the same distribution of Internalized Psychopathology (20\%) and Mixed Psychopathology (30\%).

\section{Nonverbal Processing Strengths}

This subtype of children demonstrated elevated scores on the Perceptual Organization Index. As well, this group demonstrated higher WIAT Numerical Operations scores compared with the GD group, although the Numerical Operations mean score was lower in comparison with the PSS and VPS groups. In comparison with the PSS group, the NPS group had significant difficulty with automated tasks, which are presumably mediated through language-related processes. This group demonstrated difficulties in VABS Communication and Daily Living Skills in comparison with the VPS group. Finally, this group was most likely of all groups to exhibit significant psychopathology on the PIC (70.4\%), with an especially high proportion showing Internalized Psychopathology (40\%).

\section{Global Deficits}

This subtype demonstrated generally lower intellectual functioning in comparison with the other three groups. In addition, this group demonstrated generally lower academic achievement. However, this pattern for significantly lower WIAT Basic Reading and Mathematical Reasoning did not remain significant when controlled for Full Scale IQ and, therefore, appears to be primarily associated with IQ level. Adaptively, this group demonstrated global deficits in VABS Communication, Daily Living Skills, and Socialization. Finally, $60 \%$ of this group demonstrated significant psychopathology on the PIC, with a higher proportion showing Internalized Psychopathology $(40 \%)$ as compared with Mixed Psychopathology $(20 \%)$, which is similar in pattern to the NPS group.

The findings from this study highlight the significance of intellectual subtypes of ID. In this case, we utilized WISC-III Index scores and interpreted the implication of the results on the primary intellectual processing strengths exhibited by each group. This is because of the importance of identifying and finding ways to utilize each individual child's strengths when helping them to optimize their neurodevelopmental potential. This habilitation principle is central to the development of positive approaches and outlets that promote skill development, socialization, and functional independence, while adjusting demands to minimize the impact of the individual's deficiencies [34]. Building on a vulnerable individual's strengths is additionally important because it promotes self-esteem and selfefficacy.

Previous research has shown that children with ID are at an increased risk for developing co-morbid psychopathology $[8,33]$. We found that $50 \%$ to $70.4 \%$ of children in this study of clinic-referred children with ID showed significant psychopathology on the PIC, with differences in psychopathology rates and types (internalized or mixed psychopathology) associated with the ID subtype. Increased risk for the development of psychopathology might be especially related to unrealistic expectations of children with ID and symbol processing strengths or language-related strengths because of their relatively well-developed academic and communicative competencies. Conversely, those children with visual-motor strengths, in particular, have additional threats to their self-esteem due to their especially limited reading and communicative competencies. In all cases, having a firm understanding of the competencies, limitations, and risks associated with children with ID is essential for the development of appropriate expectations and supports, which will reduce the development of secondary psychopathology.

In addition, it should be useful to extend academic and associated intervention strategies to children with mild ID that are currently associated with children with similar patterns of learning disabilities. In doing so, parents, educators, and clinicians will be able to focus on the individual strengths of children with Intellectual Disabilities and structure their interventions, environment, and expectations accordingly. This will 
reduce the likelihood that these children will develop future psychopathology due to inadequate consideration of their special developmental needs and competencies and increase the likelihood that they will learn to effectively utilize their strengths.

In summary, the results of the current investigation serve to broaden our understanding of children with ID. Specifically, the results suggest that a classification such as mild ID is overly simplistic and that there might be benefits to elucidating the different cognitive processing strengths and weaknesses and other characteristics that children with mild ID may possess. In addition, this study provides compelling evidence that identified subtypes of children with mild ID could be responsive to appropriately modified specialized interventions and remedial approaches for children with similar patterns of LD (e.g., step-by-step verbal selfguidance training for children with verbal processing strengths). The results of this study also support and validate cluster analysis as a useful statistical tool for identifying subtypes of children with ID, as has been previously demonstrated in other studies with LD and normal functioning child populations (e.g., 11, 24, 25]).

\section{Limitations}

Despite the findings of the current study, several limitations and directions for future research were noted. First, additional investigations are required to determine the academic, adaptive behavior, and psychological adjustment characteristics associated with the identified ID for further external validation utilizing additional measures, including the WISC-IV, to increase our understanding of the significance of the identified intellectual subtypes. Next, due to the significant comorbidities of other conditions such as Fetal Alcohol Syndrome, Autism, or Attention Deficit Hyperactivity Disorder, additional studies that include a larger sample of children with these conditions would be able to determine the influences of these conditions to a greater extent. Further, while differences related to parental drug use and poverty were noted in the sample, the purpose and scope of this paper prevented these trends from being further explored. As such, future research that examines the potential effect of these variables within this population would be beneficial. As well, in order to highlight the cognitive strengths noted in the current study, additional research that utilizes a high functioning control group would also be beneficial. Finally, this study points to areas for future research needed to determine additional associated attributes of specific intellectual subtypes of ID, including most appropriate academic interventions, needed treatment approaches, and guidelines for effective parenting.

\section{REFERENCES}

[1] Konold TR, Glutting JJ, McDermott PA, Kush JC, Watkins MM. Structure and diagnostic benefits of a normative subtest taxonomy developed from the WISC-III standardization sample. J Sch Psychol 1999; 37: 29-48. http://dx.doi.org/10.1016/S0022-4405(98)00024-7

[2] Saunders C, Casey J, Jones D. Patterns of WISC-III performance in a heterogeneous neuropsychological sample. Clin Neuropsychol 2001; 15: 259.

[3] Waxman RS, Casey JE. Empirically derived abilityachievement subtypes in a clinical sample. Child Neuropsychol 2006; 12: 23-38.

http://dx.doi.org/10.1080/09297040500205561

[4] American Psychiatric Association. Diagnostic and statistical manual of mental disorders, (4th ed., text revision). Washington, DC: Author 2000.

[5] Wetherby AM, Yonclas DG, Bryan AA. Communicative profiles of preschool children with handicaps: Implications for early identification. J Speech Hear Disord 1989; 54: 148-58.

[6] Kasari C, Freeman SFN, Bass W. Empathy and response to distress in children with Down syndrome. J Child Psychol Psychiatry 2003; 44: 424-31. http://dx.doi.org/10.1111/1469-7610.00132

[7] Wolff PH, Gardner J, Paccia J, Lappen J. The greeting behavior of fragile X males. Am J Ment Retard 1989; 93: 406-11.

[8] Ralston MB, Fuerst DR, Rourke BP. Comparison of the psychosocial typology of children with below average IQ to that of children with learning disabilities. J Clin Exp Neuropsychol 2003; 25: 255-73.

http://dx.doi.org/10.1076/jcen.25.2.255.13645

[9] Hodapp RM, Leckman JF, Dykens EM, Sparrow SS. K-ABC profiles in children with fragile $X$ syndrome, Down syndrome and nonspecific mental retardation. Am J Ment Retard 1992; 97: $39-46$

[10] Wechsler D. Wechsler intelligence test for children-third edition. San Antonio, TX: The Psychological Corporation 1991.

[11] Donders J. Cluster subtypes in the WISC-III standardization sample: Analysis of factor index scores. Psychol Assess 1996; 8: 312-18. http://dx.doi.org/10.1037/1040-3590.8.3.312

[12] American Association on Mental Retardation. Mental retardation: Definition, classification, and systems of supports. Washington, DC 2002.

[13] Sparrow SS, Balla DA, Cichetti DV. Vineland adaptive behavior scales. Circle Pines, MN: American Guidance Service 1984.

[14] Carpentieri S, Morgan SB. Adaptive and intellectual functioning in autistic and nonautistic retarded children. $J$ Autism Dev Disorder 1996; 26: 611-20. http://dx.doi.org/10.1007/BF02172350

[15] Liss M, Harel B, Fein D, et al. Predictors and correlates of adaptive functioning in children with developmental disorders. J Autism Dev Disord 2001; 31: 219-30. http://dx.doi.org/10.1023/A:1010707417274

[16] Bacon A, Fein D, Morris R, Waterhouse L, Allen D. The response of autistic children to the distress of others. J Autism Dev Disord 1998; 28: 129-42. http://dx.doi.org/10.1023/A:1026040615628 
[17] Vig S, Jedrysek E. Adaptive behavior of young urban children with developmental disabilities. Ment Retard 1995; 33: 90-98.

[18] Wechsler D. Wechsler individual achievement test. San Antonio, TX: The Psychological Corporation 1992.

[19] Rourke BP Fuerst DR. Learning disabilities and psychosocial functioning: A neuropsychological perspective. New York: Guilford Press 1991.

[20] Nunn RJ, Tonge BJ, Einfeld SE. Psychopathology subtypes in young people with mental retardation: A cluster analytical study. J Intellect Disabil Res 2000; 44: 864.

[21] American Association on Intellectual and Developmental Disabilities. Intellectual disability: Definition, classification, and systems of supports (Eleventh edition). Washington, DC 2010.

[22] Kanaya T, Scullin MH, Ceci SJ. The Flynn Effect and U.S. policies: The impact of rising IQ scores on American society via mental retardation diagnoses. Am Psychol 2003; 58: 77890.

http://dx.doi.org/10.1037/0003-066X.58.10.778

[23] Morris R, Blashfield R, Satz P. Neuropsychology and cluster analysis: Potentials and problems. J Clin Neuropsychol 1981; 3: $79-99$.

http://dx.doi.org/10.1080/01688638108403115

[24] Fuerst DR, Rourke BP. Psychosocial functioning of children: Relations between personality subtypes and academic achievement. J Abnorm Child Psychol 1993; 21: 597-60. http://dx.doi.org/10.1007/BF00916445

[25] Donders J, Warschausky S. WISC-III factor index score patterns after traumatic head injury in children. Child Neuropsychol 1997; 3: 71-78. http://dx.doi.org/10.1080/09297049708401369

[26] Hurley G, Levinson E. Profiles of learning disability subtypes in a retrospective sampling of adolescents' scores on the woodcock-johnson psycho-educational battery-revised. Psychol Rep 2002; 90: 767-70.

http://dx.doi.org/10.2466/pr0.2002.90.3.767

[27] Koushik NS, Saunders CD, Rourke BP. Patterns of cognitive functioning in a referred sample of preschool children. Can $J$ Sch Psychol 2007; 22: 94-107. http://dx.doi.org/10.1177/0829573507301398

[28] Rourke BP, Finlayson MAJ. Neuropsychological significance of variations in patterns of academic performance: Verbal and visual-spatial abilities. J Abnorm Child Psychol 1978; 6: 121-33. http://dx.doi.org/10.1007/BF00915788

[29] Saunders C, Strang J, Jones D, Goertzen L. Subtypes of WIAT performance in a heterogeneous neuropsychological sample. Appl Multivariate Res 2006; 12: 95-105.

[30] Wiegner S, Donders J. Performance on the Wisconsin Card Sorting Test after traumatic brain injury. Assess 1999; 6: 179187. http://dx.doi.org/10.1177/107319119900600205

[31] Jones DA, Drummond C, Saunders CD, Strang JD. Creating an empirical typology: A review of cluster analysis and other classification techniques. Appl Multivariate Res 2006; 12: 7393.

[32] Morris RD, Fletcher JM. Classification in neuropsychology: A theoretical framework and research paradigm. J Clin Exp Neuropsychol 1988; 10: 640-58. http://dx.doi.org/10.1080/01688638808402801

[33] Emerson E. Prevalence of psychiatric disorders in children and adolescents with and without intellectual disability. J Intellect Disabil Res 2003; 47: 51-58. http://dx.doi.org/10.1046/j.1365-2788.2003.00464.x

[34] Strang JD, Saunders CD, Casey JE. Enhancing clinical effectiveness through partnerships: A neuropsychological model. Ont Psychol 2002; 34: 6-7.

\section{DOI: http://dx.doi.org/10.6000/2292-2598.2013.01.01.6}

(C) 2013 Taylor et al.; Licensee Lifescience Global.

This is an open access article licensed under the terms of the Creative Commons Attribution Non-Commercial License (http://creativecommons.org/licenses/by-nc/3.0/) which permits unrestricted, non-commercial use, distribution and reproduction in any medium, provided the work is properly cited. 